\title{
Hail Detector and Forecaster ArtAr-HDF
}

\author{
Artashes K. Arakelyan, Vanik V. Karyan, Maria K. Arakelyan \\ ECOSERV Remote Observation Centre Co. Ltd., Yerevan, Armenia \\ Email: arakelyanak@yahoo.com, ecoservroc@yahoo.com
}

How to cite this paper: Arakelyan, A.K., Karyan, V.V. and Arakelyan, M.K. (2020) Hail Detector and Forecaster ArtAr-HDF. Agricultural Sciences, 11, 966-982. https://doi.org/10.4236/as.2020.1111063

Received: September 24, 2020

Accepted: November 9, 2020

Published: November 12, 2020

Copyright (c) 2020 by author(s) and Scientific Research Publishing Inc. This work is licensed under the Creative Commons Attribution International License (CC BY 4.0).

http://creativecommons.org/licenses/by/4.0/

\section{(c) (i) Open Access}

\begin{abstract}
This article describes a new and low-cost microwave passive sensor for hail prediction (forecasting) and detection developed in Armenia, which can be used to implement fully autonomous and automatically functioning hail protection of locally limited or large agricultural and urban areas in order to prevent, suppress or catch hail in traps. The article also presents the results of measurements of the intrinsic emission characteristics of water and ice, rain and hail clouds, carried out in laboratory and field conditions in the Ku-band of radio frequencies. The results obtained showed that the intrinsic emission of a hail cloud in the Ku-band of radio frequencies differs significantly from the intrinsic emission of a rain cloud. The presented results show that indeed the radar is not very suitable for the timely detection and determination of hail with a high probability, which is very important for the timely starting up of anti-hail protection means. On the contrary, radiometers (passive microwave sensors) can become an effective sensing tool for timely detection and recognition of hail with a high probability of long-range approaches up to $\sim 12-15 \mathrm{~km}$.
\end{abstract}

\section{Keywords}

Hail, Hail Detection, Hail Forecasting, Hail Prevention and Suppression, Hail Trapping, Brightness Temperature, Microwave Radiometer

\section{Introduction}

Along with the existing difference between world population growth rates and food production, a continuous increase in environmental pollution and environmental degradation, diseases such as COVID-19 or more serious can cause hunger on the planet. Therefore, in order to partially overcome the inevitable famine on the planet it is necessary primarily to solve the problem of lossless preservation of the crop. The main obstacles to solving the problem of lossless preservation of the crop are natural disasters such as droughts, floods, storms 
(strong winds) and tornadoes, earthquakes and volcanic eruptions, early or late frosts, bad weather (continuous rains) during sowing and harvesting, hail and rainfall, etc. Many of these disasters are not subject to man at all and cannot be controlled by him. And only hail and rain in some cases can be controlled. The only problem is the effectiveness and cost of such management.

A detailed description of the flaws of existing methods and stations of anti-hail protection and the advantages of new, recently developed methods and networks of anti-hail protection and their application for anti-hail protection of restricted or vast agricultural and urban areas are presented in [1] [2] [3] [4]. Despite the effectiveness of these methods which aroused wide interest and discussions among interested users and specialists, their use is temporarily delayed due to the lack of a prototype of hail detector-forecaster. Thus, the development of a cheap hail detector-forecaster and its field testing is an urgent and important task for the agriculture, the economy of the state and nature.

In this article a new and cheap sensor for predicting (forecasting) and early detecting hail at a distance up to $12-15 \mathrm{~km}$ is described, and some experimental results of its application are presented. The developed detector-forecaster can be successfully used for implementation of fully autonomous and automatically functioning anti-hail protection of locally limited or vast agricultural and urban areas in order to prevent, to suppress or to capture hail in trap areas.

\section{Hail Detector-Forecaster ArtAr-HDF}

ArtAr-HDF was developed on the basis of $\mathrm{Ku}$-band $(\sim 10 \mathrm{GHz})$ satellite dish antenna with LNB (low noise block downconverter). The block diagram of ArtAr-HDF entirely corresponds to the block diagram of the hail detector-alerter described in [5]-[10] in detail. It comprises a parabolic antenna (a dish), a radiometric (microwave) receiver (including LNB), a compensation device, a multi-level thresholder, a warner and a power supply. ArtAr-HDF can operate at any polarization of observation, vertical or horizontal. It is the cheapest hail detector-forecaster, since does not comprise a self-controlled and automatically functioning scanner, a remote warning device and other control means. Home-made work prototypes of ArtAr-HDF with $60-\mathrm{cm}$ and $100-\mathrm{cm}$ dish antennas and LNB set on manually controlled scanners are shown in Figures 1-3 and in Figures 4-6, respectively. ArtAr-HDF has two outputs, more sensitive and less sensitive, the signals of which can be recorded by a recorder, simultaneously (Figure 6). The sensitivity of the radiometric receiver of ArtAr-HDF at 1s integration time is better than $0.5 \mathrm{~K}$. The spatial resolution of ArtAr-HDF depends on the angle of observation $\vartheta$ and the altitude $h$ of the cloud. It can be approximately estimated by the following equation:

$$
d \approx \frac{\lambda \cdot h}{D \cdot \sin \psi}
$$

where $d$ is a diameter of the cross section (footprint) of the antenna's main beam at the altitude $h, D$ is the dish's diameter, $\lambda$ is the radio wavelength and 


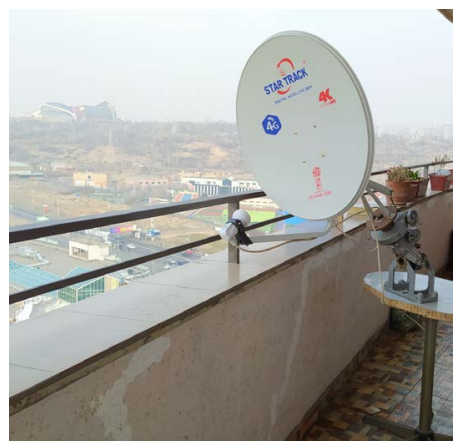

Figure 1. Ku-band, $60 \mathrm{~cm}$ dish antenna and LNB of ArtAr-HDF are installed on mechanical scanner at a height of $52 \mathrm{~m}$ from the ground in Yerevan and directed West, South-West to measure sky emission and solar emission during sunset.

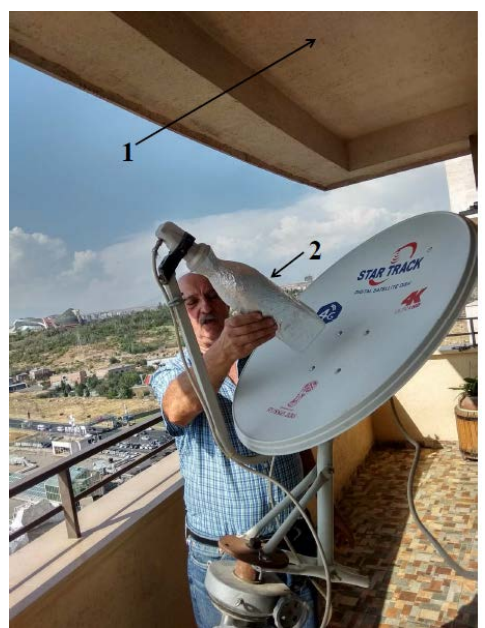

Figure 2. Calibration facilities of ArtAr-HDF in the city of Yerevan 1-Concrete ceiling (Gray Body) with an emissivity of $\sim 0.95,2$ - Black Body with an emissivity of $\sim 0.974$.

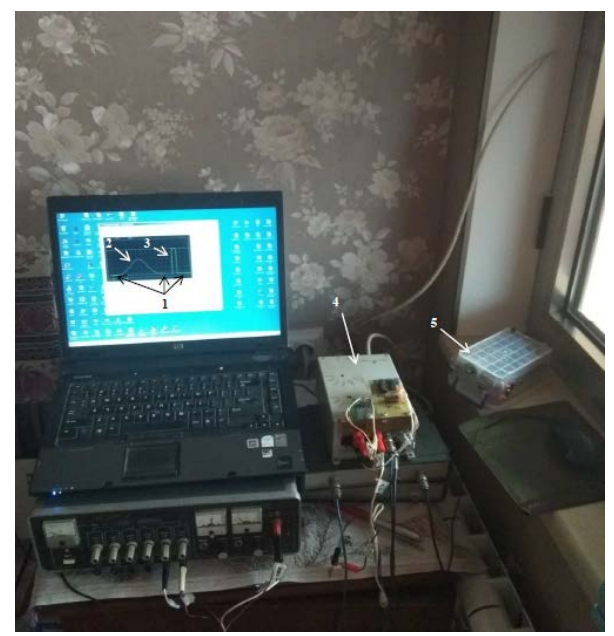

Figure 3. First prototype of ArtAr-HDF during solar microwave emission measurements in light clouds sky during sunset in Yerevan 9 January, 2020. 1-Light cloudy sky, 2-The sun during sanset (the maximum is $\sim 160 \mathrm{~K}$ ), 3-Gray Body with an emissivity of $\sim 0.95$ at $+2^{\circ} \mathrm{C}$ (the mean is $\sim 261 \mathrm{~K}$ ), 4-First prototype of ArtAr-HDF, 5-Third prototype of ArtAr-HDF. 


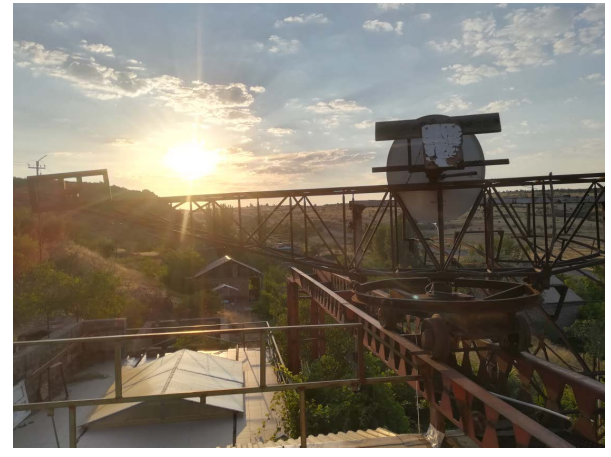

Figure 4. Ku-band, $100 \mathrm{~cm}$ dish antenna and LNB of ArtAr-HDF are installed on mechanical scanner at a height of $10 \mathrm{~m}$ from the ground in experimental site of ECOSERV ROC Co. Ltd. near the Village of Ujan and directed East to measure sky emission and solar emission during sunrise.

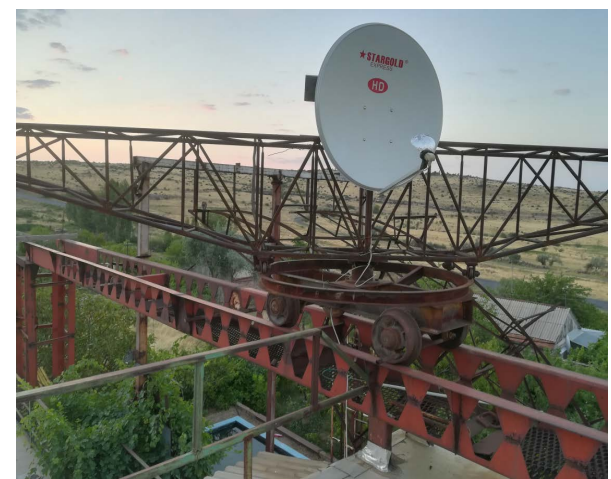

Figure 5. Ku-band, $100 \mathrm{~cm}$ dish antenna and LNB of ArtAr-HDF are installed on mechanical scanner at a height of $10 \mathrm{~m}$ from the ground in experimental site of ECOSERV ROC Co. Ltd. near the Village of Ujan and directed West to measure sky emission and solar emission during sunset.

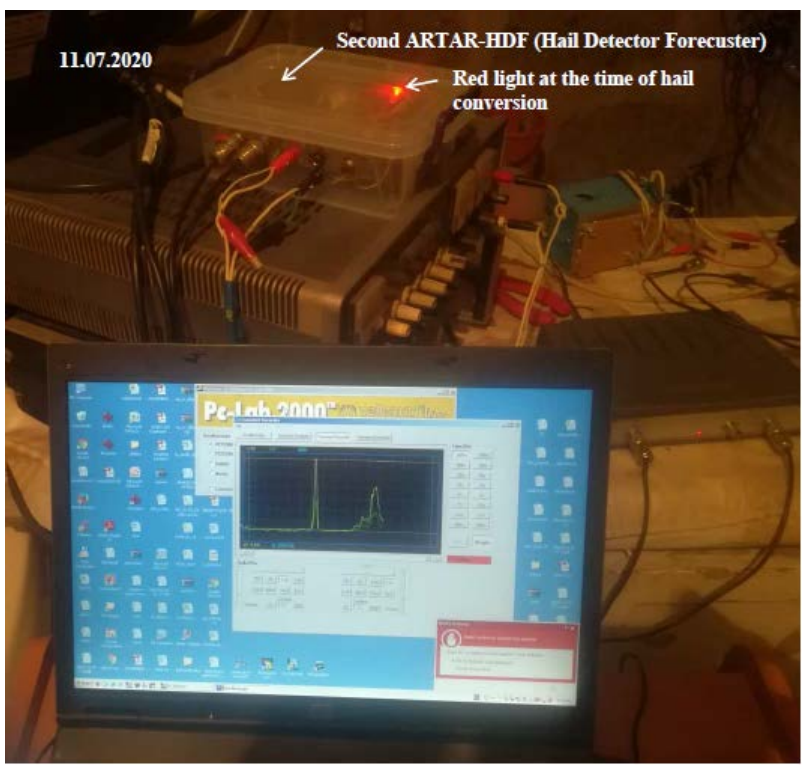

Figure 6. Second prototype of ArtAr-HDF during measurements solar and hail cloud microwave emissions in the experimental site of ECOSERV ROC Co. Ltd. 
$\psi=90^{\circ}-\vartheta$ is the grazing angle of sensing. The far zone of ArtAr-HDF is defined as

$$
r_{z} \approx \frac{D^{2}}{\lambda}
$$

In some cases, a dish is not necessary and ArtAr-HDF can only include a radiometric receiver. In such cases, in Equation (1) and in Equation (2), it is necessary to replace $D$ by $D_{f}$ where $D_{f}$ is a diameter of LNB feed horn which is approximately equal $4.5-4.7 \mathrm{~cm}$.

\section{Testing and Trial of ArtAr-HDF}

Testing and trials of all three developed prototypes of ArtAr-HDF were carried out in quasi-field and field conditions in the city of Yerevan and at the experimental site (test site) of ECOSERV ROC Co. Ltd., near the village of Ujan, where hail is more likely. First experiments had the aims to understand the behavior of developed radiometers in field conditions during long time measurements of sky emission, to study fluctuations and trends of their output signals.

For such studies, an external and stable microwave emission source is required, which would make it possible to assess the sensitivity of the radiometers and their performance. Only the sun could be such a source in field conditions. However, daily capture of the sun by a narrow beam of a satellite dish is a very difficult task due to the fact that it is constantly changing its position in the sky, and it is very difficult to track these changes by mechanical scanners. Therefore, it was only possible to work with the sun for a couple of days with taking into account the fact that the maximum emission would varied from day to day, depending on how close or far from the central axis the sun crossed the main beam of antenna on the day of measurement. This is obvious if to compare data shown in Figure 3 and Figure 7 which were measured at the same time and from the same position and direction with a difference of 3 days only. So, the sun cannot be used for each day calibration of measured data. Thus, to calibrate the results of measurements carried out in the city of Yerevan, a dry, scabrous and painted balcony ceiling of concrete was used as a Gray Body (see Figure 2) with an experimentally estimated steady emissivity about 0.95 . Calibration of measurements carried out in ECOSERV ROC's test site was performed through the horn input of LNB by a developed so-called Black Body with an emissivity of $\sim 0.974$ which is worn directly over the LNB plastic cap as shown in Figure 2. A power of intrinsic emission in radio frequencies of any body is directly proportional to its brightness temperature $T_{B}$ which is determined by the following equation

$$
T_{B}=\chi \cdot T_{0}
$$

where $\chi$ is the emissivity of the body, and $T_{0}$ is its physical temperature in Kelvin. For our experiments, as physical temperatures of Gray and Black Bodies the ambient or surrounding air temperatures is used. 


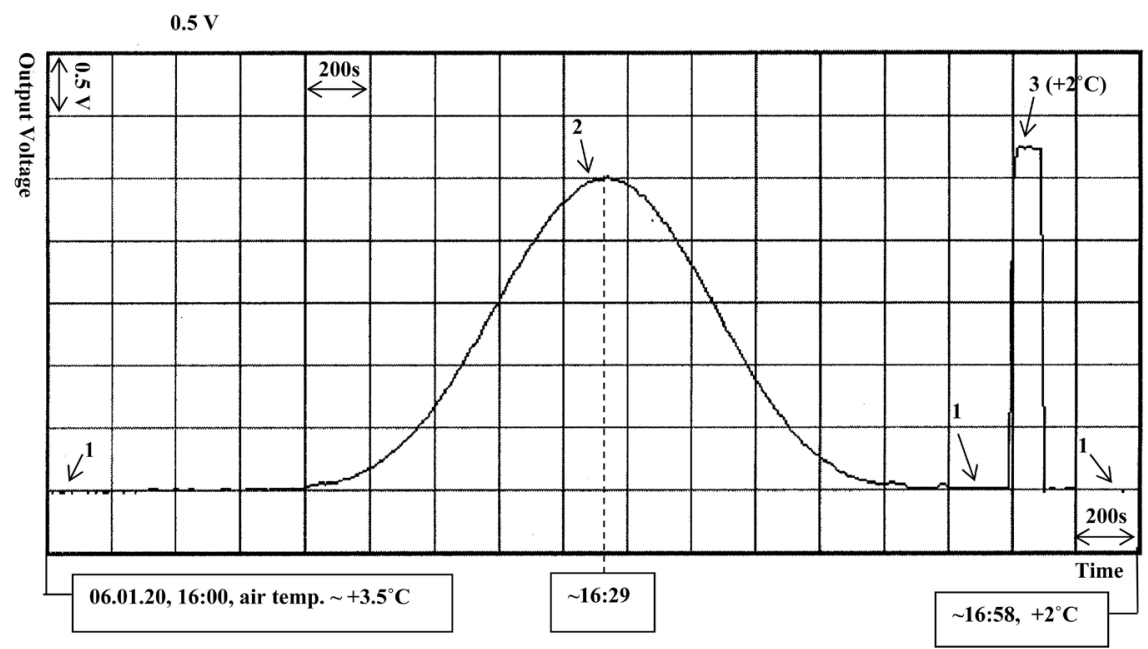

Figure 7. A split chart of the record of the sun emission during sunset in Yerevan 06. January, 2020. 1-Overcast (somber) sky, 2-The sun during sunset (the maximum is $\sim 237.5 \mathrm{~K}$ ), 3-Dry, Gray Body with an emissivity of $\sim 0.95$ at $+2^{\circ} \mathrm{C}$ (the mean is $\sim 261 \mathrm{~K}$ ).

Thus, the results of measurements of solar microwave emission in different weather conditions can be used to show that ArtAr-HDF has quite good operational performance and satisfactory sensitivity.

\section{The Results of Laboratory and Field Measurements}

\subsection{The Results of Laboratory Testing of ArtAr-HDF}

To show and convince that ArtAr-HDF can serve as a hail detector and can be used as a hail forecaster (predictor), it is necessary to experimentally confirm that when water droplets in clouds are transformed (converted) into hail pellets then the brightness temperature of the cloud increases significantly. Although, this statement has been theoretically proven in [3], however, most (if not all) potential users, experts and consumers want to see this firsthand. Directly solving this problem is practically beyond the power of even large research institutions (not to mention a lone researcher), since the transient process of water drops or water vapor into hail is not such a frequent phenomenon in nature, and it will be necessary to build many observation stations in various places over a wide area and equip them with microwave passive sensing devices and meteorological monitoring equipment and wait a long time to capture and record the event. Therefore, it was decided to carry out indirect laboratory studies of microwave features of freezing water and ice in controlling conditions, the results of which could be used to explain the phenomenon under study. With this purpose an experimental stand was built in semi-open space (outside the building) at a height of $52 \mathrm{~m}$ from ground (as shown in Figure 8) to decrease influence of walls and surrounding objects intrinsic emissions and to exclude a possible specular (mirror) reflection of ambient intrinsic emission through water and ice surfaces during measurements. Laboratory measurements were carried out by 


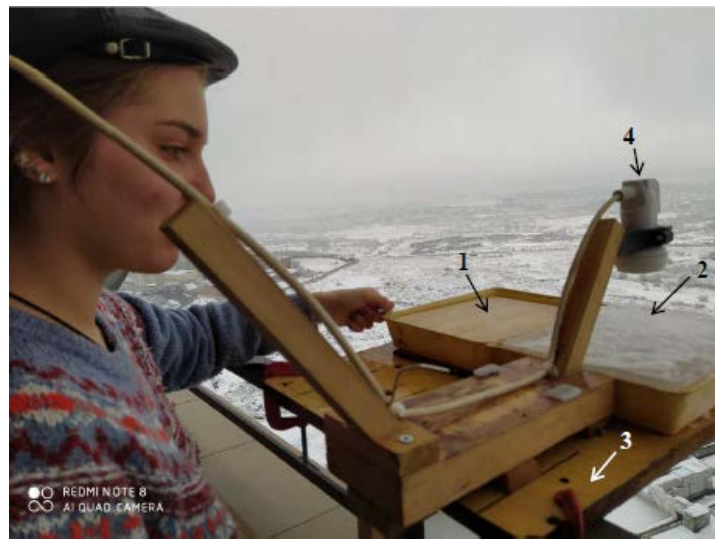

Figure 8. Experiments with water and ice in plastic trays placed on a wooden stand. 1-A plastic tray with water of $\sim 4 \mathrm{~cm}, 2-$ A plastic tray with rectangular rough ice layer of $\sim 4.5$ $\mathrm{cm}$ at $\sim 4^{\circ} \mathrm{C}, 3-\mathrm{A}$ wooden stand, $4-\mathrm{LNB}$.

the radiometric receiver (including LNB) of the first prototype of ArtAr-HDF, without the dish. In this case, the condition for sensing in the far zone and the condition for the complete positioning of the beam footprint of the feed horn of LNB within the surface of the investigated substance in the tray were ensured. LNB was directed to water or ice surface in trays under the angle of incidence $\sim 12^{\circ}$.

Initially, experiments were carried out with trays installed on wooden stand and filled with water or frozen ice (see Figure 8). Trays were always installed in the same place and probed (observed) by LNB from the same position under the same angles of incidence and azimuth. For calibration of data of measurements LNB sometimes was directed to the same place of the balcony dry ceiling (Gray Body) with an emissivity of $\sim 0.95$ (see Figure 2). At any changes in the course of the experiment, the time of the corresponding change and air temperature were recorded, and since, it was almost constant during the day, the air temperature can be used as the physical temperature of the environmental objects like ceiling, for instance.

In Figure 9 a strip chart of the record is presented of microwave emissions of freezing water and hardening ice of last two days of multi-day experiment. The level of the signal at the beginning of this chart and labeled 1 corresponds to a rectangular and smooth ice layer of $\sim 4.5 \mathrm{~cm}$ in tray and frozen in freezer to $-18^{\circ} \mathrm{C}$. The levels of the signal labeled 2 correspond to a rectangular and rough ice layer of $\sim 4.5 \mathrm{~cm}$ in tray and frozen by snow to $\sim-4^{\circ} \mathrm{C}$ (see Figure 8 ). The roughness on this ice layer were appeared after melting of snow layer (snowpack) $\sim 4 \mathrm{~cm}$ thick covered the surface of the tray with a smooth ice layer and freezing melted water drops on ice. The levels of the signal labeled 3 correspond to smooth water surface at $\sim-0.5^{\circ} \mathrm{C}$. The curve labeled 4 corresponds to an emission of freezing smooth water surface which are transformed into ice. The level of the signal labeled 5 corresponds to smooth water surface at $\sim+43.5^{\circ} \mathrm{C}$ which at the end of the experiment is cooled to $\sim 0^{\circ} \mathrm{C}$. Therefore, the difference 


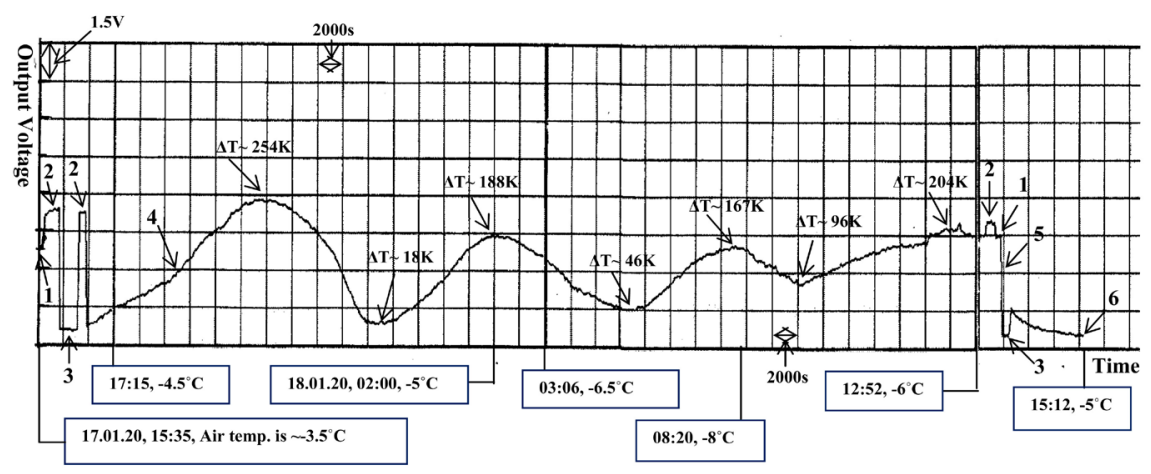

Figure 9. A strip chart of the record of microwave emissions of freezing water and hardening ice. 1 -Rectangular and smooth ice layer of $\sim 4 \mathrm{~cm}$ at $\sim 18^{\circ} \mathrm{C} ; 2-$ Rectangular and rough ice layer of $\sim 4 \mathrm{~cm}$ at $\sim-4^{\circ} \mathrm{C}, 3-\mathrm{A}$ plastic tray filled with water of $\sim 4 \mathrm{~cm}$ at $\sim-0.5^{\circ} \mathrm{C}$, 4-Freezing water of $\sim 4 \mathrm{~cm}$ in a plastic tray, 5-A plastic tray filled with water of $\sim 4 \mathrm{~cm}$ at $\sim+43.5^{\circ} \mathrm{C}, 6-$ Water cooled to $0^{\circ} \mathrm{C}$ in plastic tray.

of the signal levels marked by 5 and 6 which is equal $\sim 43.5$ Kelvin can be used as a calibration level (signal) to calibrate all measured data. The results in Figure 9 show that the level of intrinsic emission of freezing water relatively to the level of unfrozen, cold water at $0^{\circ} \mathrm{C}$ periodically changes. The difference between these emission levels, hereinafter referred to as thermal contrasts $\Delta T$, periodically increases, even reaching a value of $254 \mathrm{~K}$, and decreases, reaching even to a minimum value of $18 \mathrm{~K}$. In Figure 9 also shown the values of these contrasts estimated at different points of the maximum and minimum levels of the curve 4 . These $\Delta T$ contrasts were estimated based on a calibration level of $43.5 \mathrm{~K}$ and correspond to the points indicated in Figure 9 by the arrows.

During further experimental studies, analysis of measured data and conditions of experiments it was found that periodical behavior of the change of thermal contrasts $\Delta T$ of freezing water was due to the fact that during the experiments the water in the tray did not completely freeze. Inside of the ice layer of the tray a cavity with cooled water was remained, enveloped in an ice shell of $1-2 \mathrm{~cm}$ walls from all sides, and the volume of this cavity changed during the day. This was due to the fact that in 2020 the winter in the city of Yerevan was warm and there were no severe frosts that could completely freeze the water of $\sim 4 \mathrm{~cm}$ in the tray. Since the weather forecast did not predict frost or cold snaps in the coming weeks for the city of Yerevan, in order to avoid problems with incomplete freezing of water and hardening of ice, it was decided to change something in the experimental procedure. Namely, the wooden stand was covered with a duralumin sheet $4 \mathrm{~mm}$ thick, and the plastic trays were replaced with duralumin trays $1.5 \mathrm{~cm}$ deep, into which water was poured about $8-9 \mathrm{~mm}$ (see Figure 10). The tray-free right side of the duralumin sheet (see Figure 10) was used to measure the specular reflection of sky emission through the duralumin sheet. A thickness 8 - $9 \mathrm{~mm}$ for a layer of water is more than enough for studying microwave emission of smooth water surface, since the depth of penetration of electromagnetic waves of $\mathrm{Ku}$ band of microwave into water is very small and is only 


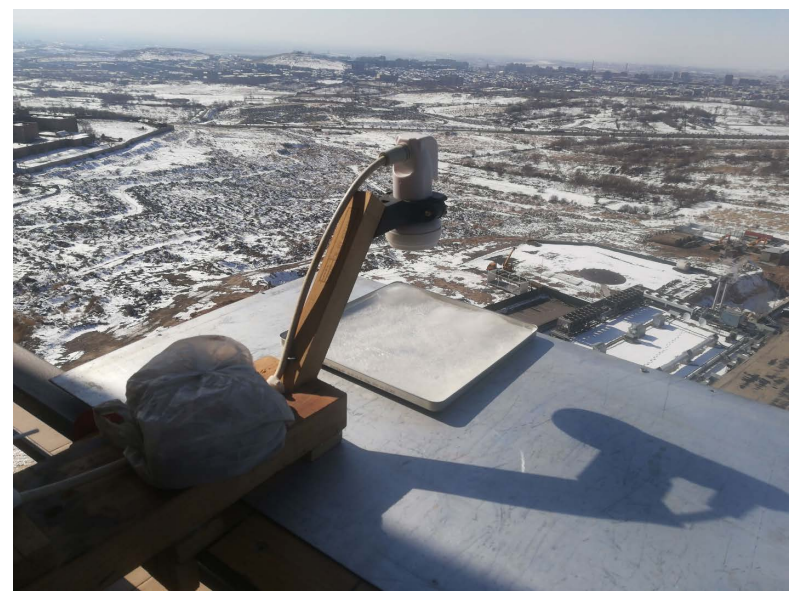

Figure 10. An experiment with a duralumin tray with frozen thin $(8 \mathrm{~mm})$ ice layer on a duralumin sheet.

a fraction of a millimeter. As for the layer of ice 8 - $9 \mathrm{~mm}$ thick, this decision was made due to the fact that pellets of hail with a diameter of $8-9 \mathrm{~mm}$ are already quite dangerous hail, and a further increase in their diameter will not significantly affect the emission characteristics of hail clouds. After all these preparations and changes, it was still managed to conduct an experiment during next couple of days with subzero air temperature and get an interesting result. Really, in Figure 11 a split chart of the record is presented of microwave emissions of freezing water and hardening ice of two consequent days of experiment, as well as sky emission and emission of the Gray Body for external calibration of data of measurements. So, the level of the signal labeled 1 corresponds to open sky emission measured by LNB directed directly to sky under elevation angle $\sim 45^{\circ}$. The levels of the signal labeled 2 correspond to an emission of sky reflected through the duralumin sheet under $10^{\circ}-12^{\circ}$. The level of the signal labeled 3 corresponds to the empty duralumin tray on the duralumin sheet. The level of the signal labeled 4 corresponds to a water layer in the duralumin tray of $\sim 8 \mathrm{~mm}$ at $\sim 0^{\circ} \mathrm{C}$. The levels of the signal labeled 4 a correspond to a water layer in the duralumin tray of $\sim 8 \mathrm{~mm}$ at $\sim 0^{\circ} \mathrm{C}$ after performed compensation.

The levels of the signal labeled 5 correspond to an emission of sky reflected through the duralumin sheet under $10^{\circ}-12^{\circ}$ after performed compensation. The levels of the signal labeled 6 correspond to an emission of transparent ice layer of $\sim 4 \mathrm{~cm}$ at $\sim-18^{\circ} \mathrm{C}$ (see Figure 12). The levels of the signal labeled 7 correspond to an emission of dry, Gray Body (a balcony ceiling) with an emissivity of $\sim 0.95$ at $\sim-4^{\circ} \mathrm{C}$ or $\sim-6^{\circ} \mathrm{C}$ of the air. The curves labeled 8 correspond to an emission of freezing water $8-9 \mathrm{~mm}$ thick in the duralumin tray. The curves labeled $8 \mathrm{a}$ correspond to an emission of hardened and drying ice of $9-10 \mathrm{~mm}$ thick in the duralumin tray. The level of the signal labeled 9 corresponds to the duralumin tray empted of ice layer after performed compensation. The level of the signal labeled 10 corresponds to the ice layer at $\sim-6.5^{\circ} \mathrm{C}$ removed from the duralumin tray 06:50 in the morning (see Figure 13). The curve labeled 11 can possibly 


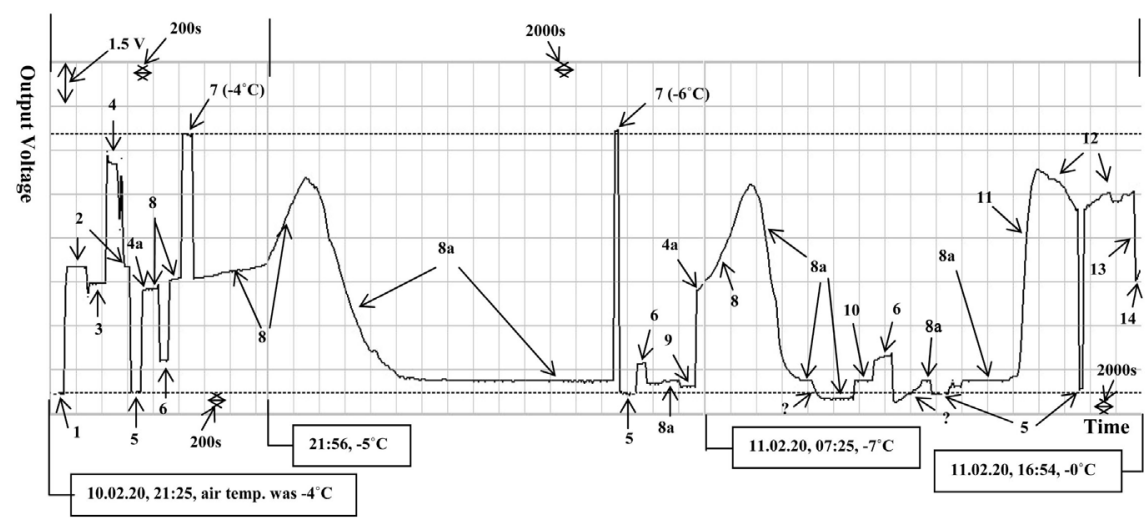

Figure 11. A strip chart of the record of emission of clear and open sky during the whole experiment. 1-Sky under $45^{\circ}, 2-$ Sky through duralumin sheet under $10^{\circ}-12^{\circ}$, 3-Empty duralumin tray, 4-A water layer in the duralumin tray of $\sim 8 \mathrm{~mm}$ at $0^{\circ} \mathrm{C}$, $4 \mathrm{a}-\mathrm{A}$ water layer in the duralumin tray of $\sim 8 \mathrm{~mm}$ at $0^{\circ} \mathrm{C}$ after compensation, 5-Sky through the duralumin sheet after compensation, 6-Rectangular, smooth and transparent ice layer of $\sim 4 \mathrm{~cm}$ at $-18^{\circ} \mathrm{C}, 7-$ Dry, Gray Body with an emissivity of $\sim 0.95$ at $-4^{\circ} \mathrm{C}$, 8 -Freezing water $8 \mathrm{~mm}$ thick in the duralumin tray, $8 \mathrm{a}-$ Hardened and drying ice of $\sim 8$ $\mathrm{mm}$ in the duralumin tray, 9-The duralumin tray empted of ice layer after compensation, 10-Ice layer of $8 \mathrm{~mm}$ (removed from the tray 06:50 in the morning) at $-6.5^{\circ} \mathrm{C}$, 11 -Possibly this is the reflection of the sun through the ice surface or from the bottom of the duralumin tray, 12-Melting of ice layer, 13-Sinking of the ice layer into the water of tray, 14-Floating layer of melted and wet ice.

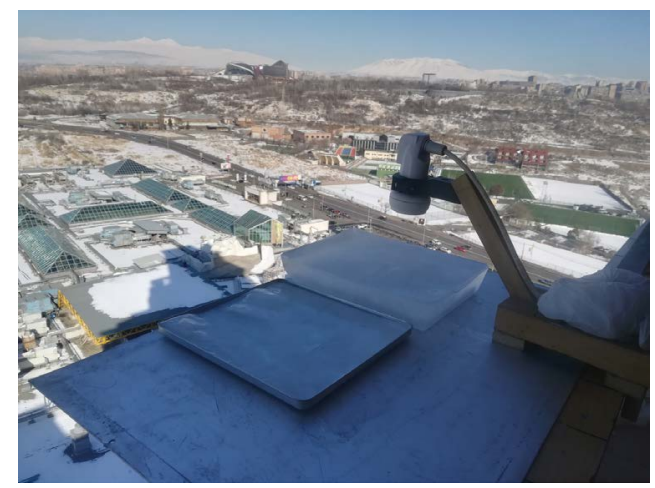

Figure 12. An experiment with $8 \mathrm{~mm}$ thin and $4.5 \mathrm{~cm}$ thick ice layers on a duralumin sheet.

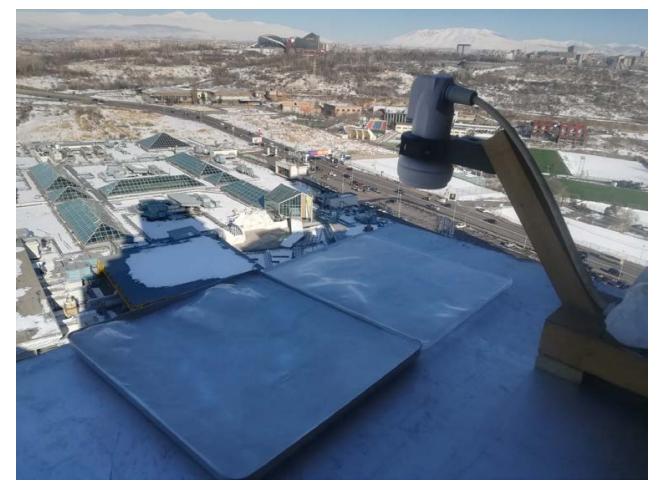

Figure 13. An experiment with two $8 \mathrm{~mm}$ thin ice layers on a duralumin sheet. 
correspond to the sun accidental specular reflection through the ice surface of the duralumin tray or through the bottom of the duralumin tray filled with hardened and dry ice layer from the left side of the tray as can be seen in Figure 10. The curves labeled 12 correspond to the ice layer in the duralumin tray melting under the lights of the sun. The curve labeled 13 corresponds to the process of sinking of melted and thin ice layer into melted water in the duralumin tray. And finally, the level of the signal labeled 14 corresponds to an emission of floating on the water surface melted and wet ice thin layer. If to use the difference between the first level of the signal labeled 7 and the level labeled 5 (a duralumin sheet after a compensation) as an external calibration signal corresponding to $\sim 255.5 \mathrm{~K}(0.95 \times 269 \mathrm{~K})$ then it will be possible to estimate approximately thermal contrasts for various points of the split chart of Figure 11. In particular, performed estimations shows that the thermal contrast between the levels labeled $4 \mathrm{a}$ (smooth water surface at $\sim 0^{\circ} \mathrm{C}$ ) and 5 is equal to $\sim 107 \mathrm{~K}$. The thermal contrast between the maximum level of the curve labeled 8 and the level labeled 5 is about $223.5 \mathrm{~K}$. The thermal contrast between the minimum level of the curve labeled 8 and the level labeled 5 is about $12.8 \mathrm{~K}$. So, the thermal contrast between fully frozen (icy) water and unfrozen water may reach up to 116.5 K (223.5 K - $107 \mathrm{~K})$.

\subsection{The Results of Field Testing of ArtAr-HDF}

Field measurements of sky emission were carried out under elevation angle of antenna beam $\sim 70^{\circ}$ at various azimuth angles. Azimuth angles of observation were chosen from the condition to exclude influence of satellite (TV) signals on radiometric receiver of ArtAr-HDF. The measurements were carried out on the background of clear sky, light cloudy or cloudy sky, excluding rain clouds. For these measurements were chosen such azimuth directions under which it was possible to catch the sun, since non-rain clouds did not in fact change output signals of ArtAr-HDF and the sun emissions during such experiments would the only signal showing that ArtAr-HDF really worked. Usually, field measurements were carried out strictly under a certain azimuth and did not change during the experiment or from experiment to experiment. However, in case if it was absolutely necessary antenna was quickly rotated from one azimuth to another that was preliminary fixed as observable direction in order to follow or catch interesting clouds. These azimuths were previously investigated in detail to ensure that they had the same background levels for clear and cloudy sky and that there is no radio interference from satellites or the environment from these directions. Further, some interesting results of field measurements of atmospheric events and phenomenon will be shown and discussed.

Figure 14 shows a split chart of the record of sky emission in cloudy (overcast) weather measured 18 March, 2020 in the city of Yerevan from the west-south-west azimuth. The signal level labeled 1 corresponds to the background of cloudy sky. The level of the signal labeled 2 corresponds to an emission 


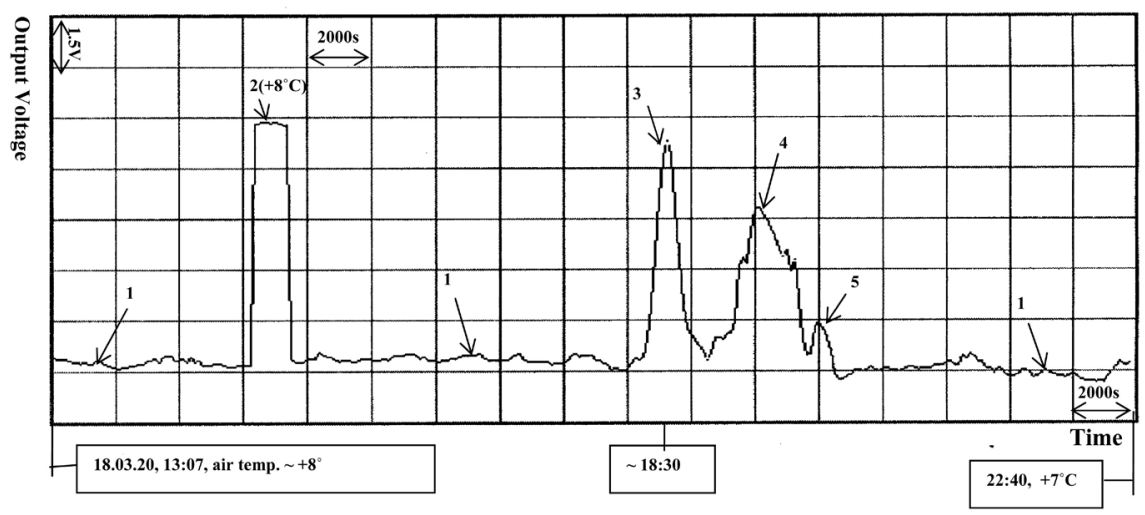

Figure 14. A strip chart of the record of emission of cloudy sky. 1-Cloudy sky, 2-Dry, Gray Body with an emissivity of $\sim 0.95$ at $+8^{\circ} \mathrm{C}, 3-$ The sun at sunset, $4-$ Hail-rain clouds, 5-Rain clouds.

of dry, Gray Body (a balcony ceiling) with an emissivity of $\sim 0.95$ at $\sim+8^{\circ} \mathrm{C}$ of the air. In accordance with this caliber, the maximum level of fluctuations of the emission of the cloudy sky before the appearance of the sun is $\sim 16 \mathrm{~K}$. The curve labeled 3 represents the sun passing through the main beam of the antenna close to the center. The maximum emission of the sun is $\sim 253.6 \mathrm{~K}$. After 15 minute of the sunset record a new atmospheric phenomenon was recorded from the same azimuth labeled 4 in Figure 14. The recorded phenomenon with the maximum emission contrast of $\sim 176 \mathrm{~K}$ is detected at a distance of $\sim 12-15 \mathrm{~km}$, at an altitude of $\sim 6 \mathrm{~km}$ and lasted for about 40 minutes or some more. It couldn't be a satellite, flying object, or UFO. Visually, it was a dark cloud. So it was most likely a hail cloud.

Very often in Armenia in early April, various atmospheric phenomenon occur, such as late frosts, snowfall, hail, etc., which partially or completely spoil initially the harvest of the affected regions. For 2020 was no exception. So, in Figure 15 a split chart of the record of sky emission for 3 uninterrupted days of April 2020 is presented. The signal level labeled 1 corresponds to the background of clear sky. The signal level labeled 2 corresponds to the background of cloudy sky. The levels of the signals labeled 2 correspond to an emission of dry, Gray Body (a balcony ceiling) with an emissivity of $\sim 0.95$ at various air temperatures indicated near the labels. The curve labeled 3 corresponds to an attempt to catch the sun. The curve labeled 4 represents the sun passing through the main beam of the antenna not so close to its central axis. The maximum emission of the sun is $\sim 171 \mathrm{~K}$. The curve labeled 5 corresponds to rain cloud with the maximum level of $\sim 83 \mathrm{~K}$. An abrupt decreasing in the signal level labeled 6 with the contrast of $\sim 21 \mathrm{~K}$ correspond to the action of cleaning the plastic cover of LNB from cold rain drops by a finger. An abrupt decreasing in the signal level labeled $6 \mathrm{a}$ with the contrast of $\sim 26 \mathrm{~K}$ corresponds to the action of cleaning the plastic cover of LNB from sprayed water drops of ambient temperature $\left(\sim 11^{\circ} \mathrm{C}\right)$ by a finger. An abrupt increasing in the signal level labeled $6 \mathrm{a}$ with the contrast 


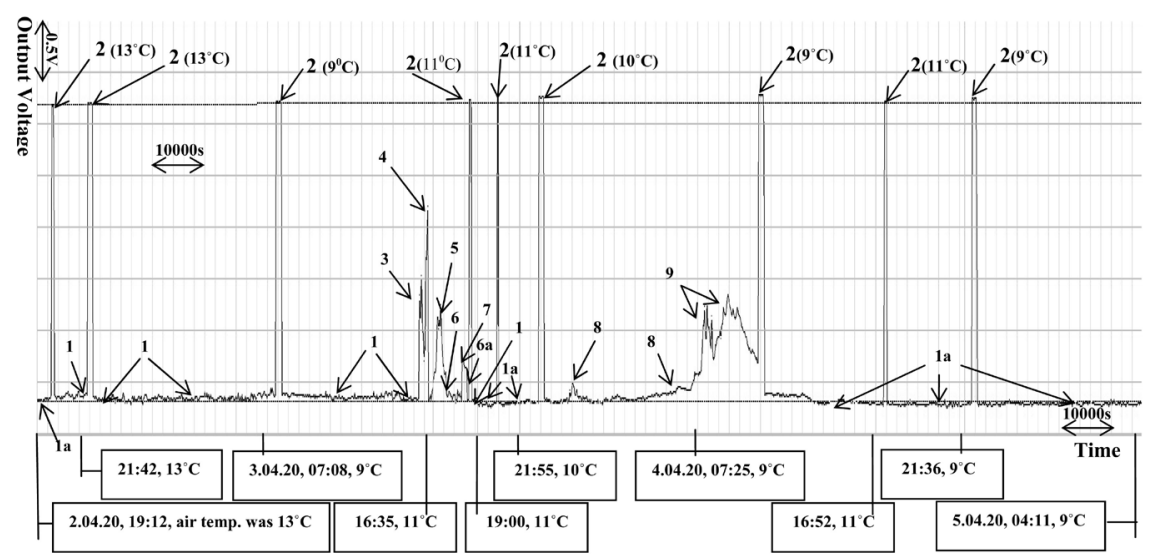

Figure 15. A strip chart of the record of emission of clear and light cloudy sky, rain and hail-snow clouds. 1-Clear sky, 1a-Light cloudiness, 2-Gray Body with an emissivity of $\sim 0.95$ (at air temperature), 3-An attempt to cutch the sun by antenna, 4-The sun at sunset, 5-Rain-hail clouds, 6-Cold raindrops were cleaned from LNB, 6a-Sprayed water drops at $11^{\circ} \mathrm{C}$ were cleaned from LNB, 7-Spraying LNB with water at $\sim 20^{\circ} \mathrm{C}$, 8-Rain clouds, 9-Rain-hail-snow clouds.

of $\sim 34 \mathrm{~K}$ corresponds to the action of spraying the plastic cover of LNB by the water at the temperature of $\sim 20^{\circ} \mathrm{C}$. The curves labeled 8 correspond to the rain and rain clouds. The curve labeled 9 with the maximum contrast of $\sim 92 \mathrm{~K}$ corresponds to the above mentioned atmospheric event which caused heavy snowfall and hail (mostly wet snow) in vast areas of the Republic of Armenia, covering by snow and ice the northern part of the city of Yerevan and many cities and villages are located north of the city of Yerevan.

The measurements carried out in clear or lightly cloudy have shown that sky emission is decreasing during the day from the morning (from 9:00-10:00) till the sunset. After the sunset sky emission is increasing and at the late evening gets its maximum value. During the night clear or lightly cloudy sky remains relatively warm with steady emission. Such behavior of sky emission can be related with the changes in ionosphere due to the sun affect. Such behaviors of sky emission for clear and lightly cloudy sky were recorded in the split charts represented in Figure 16 and Figure 17, respectively. In Figure 16 a split chart of the record of clear sky emission carried out uninterruptedly during $~ 22$ hours in the city of Yerevan. On this day, two unusual incidents were recorded: a strong wind that raised dust and fire with raised smoke. Now in more detail regarding Figure 16. The signal level labeled 1 corresponds to the background of clear and sunny sky. The curve labeled 2 corresponds to the strong wind raised a curtain of dust covered the horizon in front of the antenna beam. The maximum contrast labeled by small arrow is $\sim 108.8 \mathrm{~K}$ and the smallest contrast labeled with the long arrow is $\sim 27.9 \mathrm{~K}$. The curve labeled 3 corresponds to a strong and vast fire due to the ignition of dry grass and other vegetation at a distance of 350 - $400 \mathrm{~m}$ from the antenna. The convective flow of warm air rose to the level of the antenna beam and above and was recorded by ArtAr-HDF before the fire 


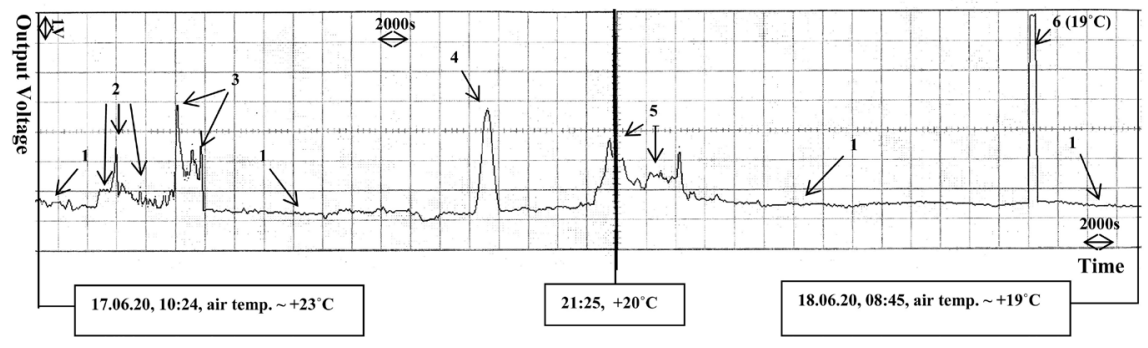

Figure 16. A strip chart of the record of emission of clear and cloudy sky and aftermath of storm and fire. 1-Clear and sunny sky, 2-A strong wind arose and a dust curtain formed in front of the antenna beam, 3-A stream of warm air and smoke appeared in front of the antenna beam due to the ignition of dried vegetation at a distance of $300 \mathrm{~m}$ far from antenna, 4-The sun at sunset, 5-Thunderstorm rain, 6-Dry, Gray Body with an emissivity of $\sim 0.95$ at $+19^{\circ} \mathrm{C}$.

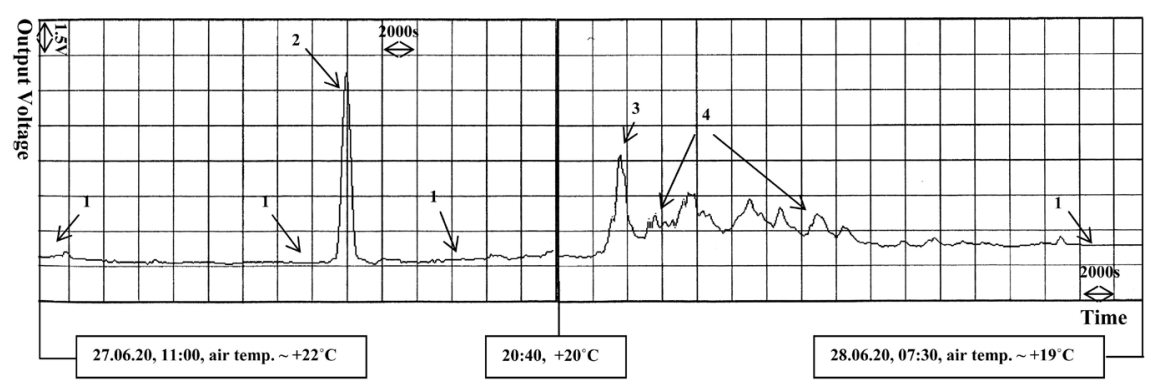

Figure 17. A Strip chart of the record of emission of lightly cloudy sky, hail and thunderstorm rain. 1-Light cloudiness, 2-The sun at sunset, 3-Probably hail cloud, 4-Thunderstorm rain.

was extinguished. The maximum contrast is $\sim 152.6 \mathrm{~K}$ and the last contrast just before the extinguishing of fire is $\sim 89.5 \mathrm{~K}$. The signal level labeled 4 corresponds to the sun emission with the maximum value $\sim 155.6 \mathrm{~K}$. The curve labeled 5 corresponds to hail-rain clouds with the first maximum contrast $\sim 96.9 \mathrm{~K}$ and with the last contrast level $\sim 76.3 \mathrm{~K}$. And at last, the signal level labeled 6 corresponds to an emission of dry, Gray Body (a balcony ceiling) with an emissivity of $\sim 0.95$ at $+19^{\circ} \mathrm{C}$. This contrast which is $\sim 277.4 \mathrm{~K}$ was used for estimating all other recorded contrasts have shown in Figure 16. In Figure 17 a split chart of the record of light cloudy sky emission carried out uninterruptedly during 20 hours in the test site of ECOSEV ROC Co. Ltd. The signal level labeled 1 corresponds to the background of light cloudy sky, and as it was mentioned above sky emission is decreasing during the day from the morning till the sunset $\sim 12.5 \mathrm{~K}$. After the sunset sky emission is increasing and close to next morning $(\sim 5: 00)$ it gets its maximum value $(\sim 18.7 \mathrm{~K})$ and remains steady till the end of the record. The signal level labeled 2 corresponds to the sun emission with the maximum value 217.4 K. During the night there were thunderstorms and rain which are labeled 3 and 4 on the split chart of the record of Figure 17, respectively. The curve labeled 3 with the maximum contrast $\sim 123.6 \mathrm{~K}$ more likely corresponds to hail clouds. The curve labeled 4 with the maximum contrast $\sim 78.6 \mathrm{~K}$ corresponds to rain and rain clouds. So, the results of field trials, selectively represented in Fig- 
ures 14-17, show that ArtAr-HDF can detect and recognize hail at the distance up to $\sim 12-15 \mathrm{~km}$ with high probability and timely warn means of anti-hail protection about coming danger.

However, for more persuasiveness of this conclusion, a direct experiment was needed, that is, not only recognition and detection of hail, but also receiving of a visual or informational confirmation about it. Unfortunately, this is a very difficult experimental task, as hail is a rare, uncontrolled and unmanaged atmospheric phenomenon, and it can be expected for years in one or two preliminary selected test areas. Therefore, the results of measurement represented below have significant value for further researches and applying. So, 11 July, 2020, closer to the night, in the west of the experimental site of ECOSERV ROC Co. Ltd., at a distance of $\sim 12 \mathrm{~km}$ and at an altitude of $\sim 5 \mathrm{~km}$, a powerful hail cloud was recorded which stirred up whole network of anti-hail protection, and, all gas generators of this area began to work without interruption in the late evening. The photo of the screen of the monitor with a split chart of transient recorder of the event of 11 July, 2020 is presented in Figure 18 (see the original in Figure 6). The record was suddenly interrupted, since, the electricity in the area has been cut off and the record was not saved in computer and therefore was lost. It's good that it was managed to take a picture of the screen right before the power outage.

In accordance with the results of the split chart of recorder represented in Figure 18, where both sensitive and insensitive outputs of ArtAr-HDF are recorded, the sun's maximum contrast is $\sim 269 \mathrm{~K}$. It means that the sun passing through the main beam of the antenna very close to the central axis. Rain clouds intrinsic emission can be $\sim 25 \mathrm{~K}$ or $58 \mathrm{~K}$ or some more. The registered maximum value of the contrast of hail intrinsic emission is $\sim 165 \mathrm{~K}$. So, the difference between hail and rain clouds intrinsic emissions is more than $100 \mathrm{~K}$.

\section{Discussion of the Results}

Thus, the results obtained during laboratory researches and presented in Figure 9 and Figure 11 showed that the nature of the behavior of the changes of intrinsic emission of water transforming (converting) into ice is steady and is repeated

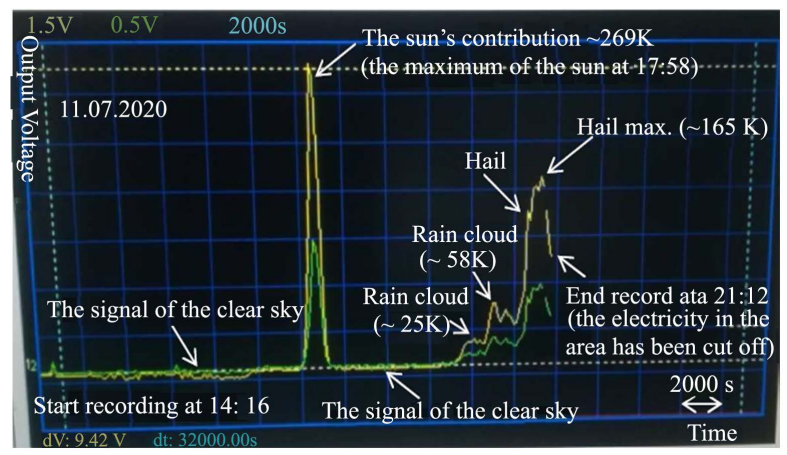

Figure 18. A sample of recording of the transient recorder on the monitor screen of the computer. 
from experiment to experiment. Frozen and icy water becomes a powerful absorber of $\mathrm{Ku}$-band radio waves. It means that frozen water droplets will reduce radar cross section of hail cloud, that is will reduce the power of backscattered radar signals. In opposite, dry and solidified (hardened) ice becomes a radio-transparent material for the same radio wave range. And, therefore, the hardened (solidified) and dried hail pellets will simply pass radio signals through themselves, thereby reducing radar cross section and reducing backscattered radar signal power. As for other ranges of radio waves, then with a decrease in the length of the radio wave (with an increase in frequency), the nature of the changes of intrinsic emissions of water and ice will probably remain the same. And with an increase in the length of the radio wave (with a decrease in frequency), the nature of changes of intrinsic emissions of water and ice possibly can change significantly. However, these issues require a separate and thorough study, which goes beyond our interests. The results obtained during laboratory experiments show once more that, indeed, radars are not suitable for the timely detection and identification (classification) of hail with a high probability, as mentioned in [3]. In contrary, radiometers can become an effective means of sensing for timely detection and recognition of hail with a high probability on long-distance approaches up to $12-15 \mathrm{~km}$. This statement is also mentioned in [3].

The results of field trials selectively represented in Figures 14-17 show that ArtAr-HDF can detect and recognize hail at the distance of up to $\sim 12-15 \mathrm{~km}$ with high probability and timely warn means of anti-hail protection about coming danger. It is due the fact that intrinsic emission of hail cloud in $\mathrm{Ku}$ band of radio frequencies differs significantly from the intrinsic radiation of a rain cloud. The difference between hail and rain clouds intrinsic emissions can be more than $100 \mathrm{~K}$. Hence, ArtAr-HDF can be successfully used for creation of global network of anti-hail protection. In Figure 19 a third prototype of ArtAr-HDF is presented which may be used without a dish if to change something in feed horn of LNB. A power consumption of ArtAr-HDF will not be more than 2 - $3 \mathrm{~W}$. A power supply of ArtAr-HDF is DC $12 \mathrm{~V}$ or $24 \mathrm{~V}$ and AC $\sim 110-220 \mathrm{~V}$.

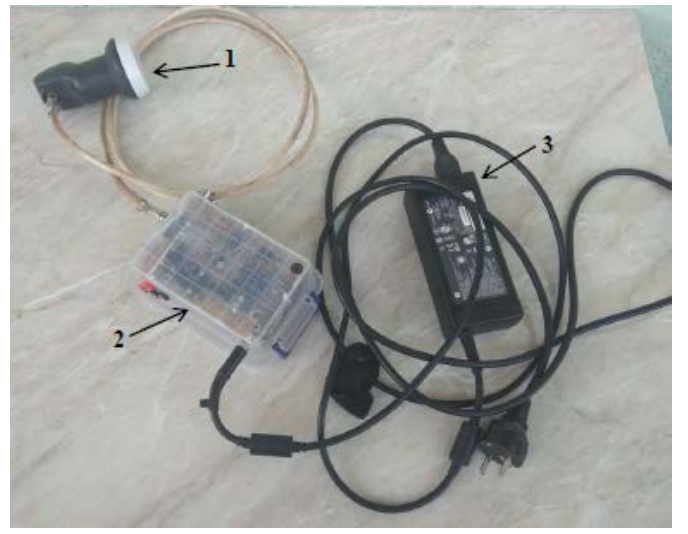

Figure 19. ArtAr-HDF. 1-LNB, 2-A low-frequency part, 3-A power supply. 


\section{Conclusion}

Thus, the results of laboratory measurements of water and ice emission characteristics have shown that radar is not suitable for the timely detection and identification of hail with a high probability which is very important for timely starting up of means of anti-hail protection. In opposite, radiometers can become an effective means of sensing for timely detection and recognition of hail with a high probability on long-distance approaches up to $\sim 12 \mathrm{~km}$. The intrinsic emission of hail cloud in $\mathrm{Ku}$ band of radio frequencies differs significantly from the intrinsic radiation of rain cloud. It is developed a cheap hail detector-forecaster which can be successfully used for timely detection and prediction of hail at a distance up to 12 - $15 \mathrm{~km}$ and for creation of a global network of anti-hail protection.

\section{Conflicts of Interest}

The authors declare no conflicts of interest regarding the publication of this paper.

\section{References}

[1] Arakelyan, A.K. (2017) A New Approach in Hail Prevention Technique for a Lo cally Restricted Area. Agricultural Sciences, 8, 559-571. https://doi.org/10.4236/as.2017.87042

[2] Arakelyan, A.K. (2017) A New Way to Implement Fully Autonomous and Automatic Large-Scale Anti-Hail Protection. Global Advanced Research Journal of Agricultural Science, 6, 361-373. http://garj.org/garjas/11/2017/6/11

[3] Arakelyan, A.K. (2018) Flaws and Advantages of Current and Prospective Anti-Hail Protection Methods, Stations and Networks (Review). Global Advanced Research Journal of Agricultural Science, 7, 366-376. http://garj.org/garjas/12/2018/7/12

[4] Arakelyan, A.K. (2019) A Way to Enhance Hail Prevention Technique and to Increase the Efficiency of Anti-Hail Protection of Unlimited Agricultural Areas. Agricultural Research \& Technology, Open Access Journal, 22, 1-4. https://juniperpublishers.com/artoaj/pdf/ARTOAJ.MS.ID.556200.pdf

[5] Arakelyan, A.K. (2013) A Method and a Network of Wide-Ranging Anti-Hail Protection. Armenian Patent \# 2769.

[6] Arakelyan, A.K. (2016) An Automated Wide-Ranging Anti-Hail Protection Method and a Network. The Patent of People's Republic of China, \# ZL2012800716222.

[7] Arakelyan, A.K. (2016) An Automated Wide-Ranging Anti-Hail Protection Method and a Network. The US Patent, \# 9491912.

[8] Arakelyan, A.K. (2017) An Automated Wide-Ranging Anti-Hail Protection Method and a Network. Canadian Patent, \# 2862959.

[9] Arakelyan, A.K. (2017) An Automated Wide-Ranging Anti-Hail Protection Method and a Network. European Patent, \# 2725893.

[10] Arakelyan, A.K. (2017) An Automated Wide-Ranging Anti-Hail Protection Method and a Network. Patent of the Russian Federation, \#2631894. 